TRANSACTIONS OF THE

AMERICAN MATHEMATICAL SOCIETY

Volume 348, Number 2, February 1996

\title{
RADIAL SOLUTIONS TO A DIRICHLET PROBLEM INVOLVING CRITICAL EXPONENTS WHEN $N=6$
}

\author{
ALFONSO CASTRO AND ALEXANDRA KUREPA
}

\begin{abstract}
In this paper we show that, for each $\lambda>0$, the set of radially symmetric solutions to the boundary value problem

$$
\begin{aligned}
-\Delta u(x) & =\lambda u(x)+u(x)|u(x)|, \quad x \in B:=\left\{x \in R^{6}:\|x\|<1\right\}, \\
u(x) & =0, \quad x \in \partial B,
\end{aligned}
$$

is bounded. Moreover, we establish geometric properties of the branches of solutions bifurcating from zero and from infinity.
\end{abstract}

\section{INTRODUCTION}

We consider the boundary value problem

$$
\begin{aligned}
-\Delta u(x) & =\lambda u(x)+u(x)|u(x)|, \quad x \in B:=\left\{x \in R^{6}:\|x\|<1\right\}, \\
u(x) & =0, \quad x \in \partial B
\end{aligned}
$$

where $\Delta$ denotes the Laplacean operator and $\lambda$ is a positive real number. Our main result is the following theorem:

Theorem 1.1. a) For each $\lambda>0$ there exist positive real numbers $j:=j(\lambda)$ and $D:=D(\lambda)$ such that if $u$ is a solution to (1.1) then $\|u\|_{\infty} \leq D$, and $u$ has at the most $j$ nodal curves.

b) Given $j$ there exists $D_{1}(j)$ such that if $\left(\lambda_{1}, u_{1}\right)$ and $\left(\lambda_{2}, u_{2}\right)$ are radial solutions to (1.1) with the property that $u_{1}$ and $u_{2}$ have $j$ nodal surfaces, $u_{1}(0)>D_{1}(j)$ and $u_{2}(0)>D_{1}(j)$ then $\lambda_{1}<\lambda_{2}$ if and only if $u_{1}(0)>u_{2}(0)$.

As shown in [5] Theorem 1.1 also holds for $N=3,4$ and 5. The proof of the case $N=6$ was defered to this paper since it requires arguments of a different kind. The main problem arises from the fact that if $N=6$ and $\lambda$ is fixed then the solutions to the initial value problem (1.4) below do not tend to zero in compact subsets of $(0,1]$ as $v(0) \rightarrow \infty$.

Motivated by the results of F. Atkinson, H. Brezis and L. Peletier (see [1]) where they studied the case $N=3,4,5,6$ in [5] we prove their conjecture that if $N=3,4$ then the problem (1.1) has only finitely many radially symmetric solutions. If $N \geq 7$ then for any $\lambda>0$ it has been proven by S. Solimini in [12] (see also [7]) that (1.1) has infinitely many radially symmetric solutions.

Received by the editors July 13, 1994 and, in revised form, February 7, 1995.

1991 Mathematics Subject Classification. Primary 35J65; 34A10.

Key words and phrases. Critical exponent, radially symmetric solutions, Dirichlet problem, nodal curves, bifurcation. 
Problems like (1.1) have been studied very intensively over the last three decades, mainly due to the fact that the well developed variational techniques do not apply because the imbedding of the Sobolev space $H_{0}^{1}(B)$ in $L^{2 N /(N-2)}(B)$ is not compact. Since 1965 it has been known (see Pohozaev [10]) that for $\lambda \leq 0$ the problem (1.1) has no nontrivial solutions. The existence of a positive solution to (1.1) for certain values of $\lambda>0$ was shown in 1982 by H. Brezis and L. Nirenberg (see [2]). They introduced a method which have influenced a number of papers and produced important results (see [6], [7] and the references therein). The main ingredient was the mountain pass theorem without the Palais Smale condition.

The proof of Theorem 1.1 depends fundamentally on the following result on the location of the zeros of the solution to

$$
\begin{aligned}
v_{d}^{\prime \prime}(t)+\frac{5}{t} v_{d}^{\prime}(t)+v_{d}(t)+2 v_{d}(t)|v(t)| & =0, \quad t \in(0,1], \\
v_{d}(0) & =1, \\
v_{d}^{\prime}(0) & =0,
\end{aligned}
$$

where $v$ is a solution to

$$
\begin{aligned}
v^{\prime \prime}(t)+\frac{5}{t} v^{\prime}(t)+v(t)+v(t)|v(t)| & =0, \quad t \in(0,1], \\
v(0) & =\frac{1}{2}, \\
v^{\prime}(0) & =0 .
\end{aligned}
$$

In fact we have:

Theorem 1.2. The zeroes of $v_{d}$ separate the zeros of $v$, and conversely.

The proof of Theorem 1.1 is based on the phase-plane analysis of the solution corresponding to a singular ordinary differential equation. We consider the initial value problem

$$
\begin{aligned}
v^{\prime \prime}(t)+\frac{5}{t} v^{\prime}(t)+\lambda v(t)+v(t)|v(t)| & =0, \quad t \in(0,1], \\
v(0) & =d, \\
v^{\prime}(0) & =0,
\end{aligned}
$$

where $d \in R$. It can be shown using the contraction mapping principle that for every $(\lambda, d)$ problem (1.2) has a unique solution $v(t):=v(t, \lambda, d)$ on the interval $[0, \infty)$ depending continuously on $(\lambda, d)$. Of course, radially symmetric solutions to (1.1) are solutions to (1.2) satisfying $v(1, \lambda, d)=0$. Because $v$ is odd in $d$, we consider only the case $d>0$. Our analysis focuses on the study of the level set $M=\{(\lambda, d): v(1, \lambda, d)=0\}$. Using the fact that the solution to (1.4) does not degenerate and some rescaling properties of $v(t)$, we show that $\left(v_{d}(1, \cdot, \cdot), v_{\lambda}(1, \cdot, \cdot)\right)$ never vanishes on $M$, where $v_{d}, v_{\lambda}$ denote the partial derivatives of $v$ with respect to $d$ and $\lambda$. Hence, $M$ is a differentiable manifold. From the results of [1] we infer that if $\Gamma$ is a connected component of $M$ then there is a radial eigenvalue $\mu_{j}$ of $-\Delta$ subject to a zero Dirichlet boundary condition, and $\nu_{j} \in\left[0, \mu_{j}\right)$ such that $\Gamma$ connects $\left(\mu_{j}, 0\right)$ with $\left(\nu_{j}, \infty\right)$. Also $\nu_{j}=0$ if and only if $j=1$. Moreover, using variants of the Sturm comparison theorem we prove that for $d$ large $v_{d}(1, \lambda, d) \cdot v^{\prime}(1, \lambda, d)>$ 0 . Thus, there exists a strictly decreasing function $s:(D, \infty) \rightarrow R$ such that $v(1, \lambda, d) \in \Gamma$ iff $d=s(\lambda)$. We combine this result with those of [1] and [5] to provide a detailed description of the branches of solutions to (1.1) bifurcating from 
zero and from infinity. Since the only connected component of $M$ that accumulates towards $\{(0, d): d>0\}$ is the one corresponding to positive solutions and since for large $d$ such a component is the graph of a strictly decreasing function we have the following corollary.

Corollary 1.3. There exists $\hat{\lambda}$ such that if $\lambda \in(0, \hat{\lambda})$ then (1.1) has a unique positive solution.

\section{Energy Estimates and Pohozaev's Identity}

The following lemma is based on Pohozaev's identity and was extensively used in [5] (see also $[3,4,11]$ ).

Lemma 2.1. If $0 \leq \tilde{t}<t$ then

$$
t^{5} H(t)-(\tilde{t})^{5} H(\tilde{t})=\int_{\tilde{t}}^{t} r^{5} \lambda u^{2}(r) d r
$$

where

$$
\begin{aligned}
H(t) & :=t\left(\frac{\left(v^{\prime}(t)\right)^{2}}{2}+\frac{|v(t)|^{3}}{3}+\lambda \frac{|v(t)|^{2}}{2}\right)+2 v(t) v^{\prime}(t) \\
& :=t E(t)+2 v(t) v^{\prime}(t) .
\end{aligned}
$$

Proof. Multiplying the equation in (1.4) simultanously by $r^{6} v^{\prime}(r)$ and $r^{5} v(r)$, integrating over $[\tilde{t}, t], 0 \leq \tilde{t}<t$, and combining the two equations, the proof of the lemma follows (for more details see [5]).

From Lemma 2.1 using the quadratic equation formula we see that

$$
\begin{aligned}
& t v^{\prime}(t)+2 v(t) \\
& = \pm \frac{1}{2} \sqrt{16 v^{2}(t)-\frac{8 t^{2}}{3} v^{3}(t)-4 \lambda t v^{2}(t)+8 t^{-4} \lambda \int_{0}^{t} r^{5} v^{2}(r) d r} \\
& := \pm \frac{1}{2} \sqrt{R(t)} .
\end{aligned}
$$

Now we define function $h$ by the following equation

$$
h(t)=-\frac{t v^{\prime}(t)}{v(t)}
$$

Using (1.2) and Lemma 2.1, as in [5], we obtain

$$
h^{\prime}(t)=\frac{2 t^{-5} \int_{0}^{t} \lambda r^{5} v^{2}(r) d r-2 t \frac{|v(t)|^{3}}{3}+t|v(t)|^{3}}{v^{2}(t)}>0 .
$$

Let $x_{1}$ denote the first zero of $v$. Since the left hand side in $(2.2)$ is positive at 0 , negative at $x_{1}$ and continuous, there exists $\hat{t} \in(0, t)$ such that $R(\hat{t})=0$. The uniqueness of $\hat{t}$ follows from (2.4). Since $v^{\prime}(0, \lambda, d)=0$ and $v(0, \lambda, d)=d$, we have

$$
t v^{\prime}(t)+2 v(t)=\frac{1}{2} \sqrt{R(t)}, \quad \text { for } t \in[0, \hat{t}]
$$

and

$$
t v^{\prime}(t)+2 v(t)=-\frac{1}{2} \sqrt{R(t)}, \quad \text { for } t>\hat{t}
$$


Lemma 2.2. Let $x_{i}$ and $x_{i+1}$ denote two consecutive zeroes of $v\left(\cdot, 1, \frac{1}{2}\right)$. Then

$$
E\left(x_{i+1}\right) \leq \frac{E\left(x_{i}\right)}{6}\left\{5\left(\frac{x_{i}}{x_{i+1}}\right)^{6}+1\right\} .
$$

Proof. From the definition of $E$ (see (2.1)) we have

$$
\frac{d E}{d r}=-\frac{5}{r} v^{\prime}(r)^{2} \text {. }
$$

Without loss of generality let $v<0$ on $\left[x_{i}, x_{i+1}\right]$. Integrating (2.7) by parts and using (1.3) we obtain

$$
\begin{aligned}
E\left(x_{i+1}\right) & =E\left(x_{i}\right)-5 \int_{x_{i}}^{x_{i+1}} \frac{v^{\prime}(r)}{r} v^{\prime}(r) d r \\
& =E\left(x_{i}\right)+5 \int_{x_{i}}^{x_{i+1}} v(r) \frac{v^{\prime \prime}(r) r-v^{\prime}(r)}{r^{2}} d r \\
& =E\left(x_{i}\right)+5 \int_{x_{i}}^{x_{i+1}} v(r) \frac{-6 v^{\prime}(r)-r v(r)-r v(r)|v(r)|}{r^{2}} d r \\
& \leq E\left(x_{i}\right)+15 \int_{x_{i}}^{x_{i+1}} v^{2}(r)\left(-\frac{2}{r^{3}}\right) d r-5 \int_{x_{i}}^{x_{i+1}} \frac{v^{2}(r)}{r} d r \\
& \leq E\left(x_{i}\right)-5 \int_{x_{i}}^{x_{i+1}} \frac{v^{2}(r)}{r} d r .
\end{aligned}
$$

On the other hand from Pohozaev's identity we have

$$
\left(x_{i+1}\right)^{6} E\left(x_{i+1}\right)=x_{i}^{6} E\left(x_{i}\right)+\int_{x_{i}}^{x_{i+1}} r^{5} v^{2}(r) d r .
$$

Combining (2.8) and (2.9) we infer

$$
\begin{aligned}
\left(1-\left(\frac{x_{i}}{x_{i+1}}\right)^{6}\right) E\left(x_{i}\right) & \geq \frac{1}{\left(x_{i+1}\right)^{6}} \int_{x_{i}}^{x_{i+1}} r^{5} v^{2}(r) d r+5 \int_{x_{i}}^{x_{i+1}} \frac{v^{2}(r)}{r} d r \\
& \geq \frac{6}{\left(x_{i+1}\right)^{6}} \int_{x_{i}}^{x_{i+1}} r^{5} v^{2}(r) d r .
\end{aligned}
$$

Using (2.10) in (2.9) we see that

$$
E\left(x_{i+1}\right) \leq E\left(x_{i}\right)\left\{\left(\frac{x_{i}}{x_{i+1}}\right)^{6}+\frac{1}{6}\left(1-\left(\frac{x_{i}}{x_{i+1}}\right)^{6}\right)\right\},
$$

which concludes the proof of the lemma. 


\section{The First Three Zeroes of $v\left(\cdot, 1, \frac{1}{2}\right)$}

In this section we estimate the location of the first three zeroes of $v\left(\cdot, 1, \frac{1}{2}\right)$.

Lemma 3.1. Let $x_{1}$ denote the first zero of $v\left(\cdot, 1, \frac{1}{2}\right)$. Then $x_{1} \leq 4.6$.

Proof. From

$$
-v^{\prime}(t)=t^{-5} \int_{0}^{t} r^{5}(1+|v(r)|) v(r) d r
$$

on $\left[0, x_{1}\right]$ we see that $v^{\prime}<0$ and

$$
-v^{\prime}(t) \geq \frac{t}{6} v(t)
$$

Hence, integrating $(3.2)$ on $[0, t]$ we obtain

$$
v(t) \leq \frac{1}{2} e^{-\frac{t^{2}}{12}}
$$

where we have also used the fact that $v(0)=\frac{1}{2}$.

Thus, from (3.3) we see that $v(3) \leq \frac{1}{4}$. On the other hand, for $t \geq 3$ we have

$$
R(t) \geq\left(16-\frac{8 t^{2}}{12}-4 t+\frac{8 t^{2}}{6}\right) v^{2}(r) \geq 0
$$

Thus $\hat{t} \leq 3$. Hence, for $t \geq 3$ from (2.6) we see that

$$
-\frac{v^{\prime}(t)}{v(t)}=\frac{2}{t}+\frac{1}{2 t v(t)} \sqrt{R(t)}
$$

In particular

$$
\begin{aligned}
-\frac{v^{\prime}(3)}{v(3)} & \geq \frac{2}{3}+\frac{1}{6} \sqrt{16-\frac{8 \cdot 9}{3 \cdot 4}-4 \cdot 3+\frac{8 \cdot 9}{6}} \\
& \geq 1.1937129 .
\end{aligned}
$$

Let $\theta$ be a differentiable function such that (see [5])

$$
\begin{aligned}
v(r) & =-\rho(r) \cos \theta(r), \\
v^{\prime}(r) & =\rho(r) \sin \theta(r), \\
\theta(0) & =0,
\end{aligned}
$$

with $\rho(r)=\sqrt{v^{2}(r)+\left(v^{\prime}(r)\right)^{2}}$. An elementary calculation shows that

$$
\theta^{\prime}(r)=\frac{\left(v^{\prime}(r)\right)^{2}+\frac{5}{r} v(r) v^{\prime}(r)+(1+|v(r)|) v^{2}(r)}{\left(v^{\prime}(r)\right)^{2}+v^{2}(r)} .
$$

Since $\tan \theta(r)=-\frac{v^{\prime}(r)}{v(r)}$ for $r \in[3,3.5]$ from (3.6) we have

$$
\begin{aligned}
\theta^{\prime}(r) & \geq 1-\frac{\frac{5}{t} \tan \theta(t)}{\tan ^{2} \theta(t)+1} \geq 1-\frac{\frac{5}{t} \tan \theta(3)}{\tan ^{2} \theta(3)+1} \\
& \geq 1-\frac{2.461314218}{t} .
\end{aligned}
$$

Integrating on $[3,3.5]$ we infer

$$
\theta(3.5) \geq .5-2.461314218 \ln \frac{3.5}{3}+\theta(3) \geq .994059 .
$$


Reiterating this argument on $[3.5,4]$ we obtain $\theta(4) \geq 1.1888816$. Similarly on $[4,4.5]$ we have $\theta(4.5) \geq 1.4852075$, and on $[4.5,4.6]$ we see that $\theta(4.6) \geq \frac{\pi}{2}$. Thus, $x_{1} \leq 4.6$. Hence the lemma is proven.

Lemma 3.2. If $x_{1}$ denotes the first zero of $v\left(\cdot, 1, \frac{1}{2}\right)$, then $x_{1} \geq 4.4$, and $\left|v^{\prime}\left(x_{1}\right)\right| \leq$ 0.134 .

Proof. Since $v$ is a decreasing function on $\left[0, x_{1}\right]$ and $v(0)=\frac{1}{2}$, from (3.1) we have

$$
-v^{\prime}(t) \leq t^{-5} \int_{0}^{t} r^{5}\left(1+\frac{1}{2}\right) \frac{1}{2} d r=\frac{t}{8} .
$$

Thus, for $t \in\left[0, x_{1}\right]$ we see that

$$
v(t) \geq \frac{1}{2}-\frac{t^{2}}{16}
$$

In particular, from (3.9) we have that $x_{1} \geq \sqrt{8}$. Furthermore, by using (3.9) in (3.1) we infer

$$
-v^{\prime}(t) \geq t^{-5} \int_{0}^{t} r^{5}\left(1+\frac{1}{2}-\frac{r^{2}}{16}\right)\left(\frac{1}{2}-\frac{r^{2}}{16}\right) d r \geq \frac{t}{8}-\frac{t^{3}}{64}+\frac{t^{5}}{2560} .
$$

Hence, on $[0, \sqrt{8}]$ we have

$$
v(t) \leq \frac{1}{2}-\frac{t^{2}}{16}+\frac{t^{4}}{256}-\frac{t^{6}}{15360} .
$$

Reiterating the argument in (3.10) we see that

$$
-v^{\prime}(t) \leq \frac{t}{8}\left(1-\frac{t^{2}}{8}+\frac{3 t^{4}}{320}-\frac{11 t^{6}}{16^{2} \cdot 36}+\frac{23 t^{8}}{16^{4} \cdot 30}\right)
$$

and

$$
v(t) \geq \frac{1}{2}-\frac{t^{2}}{16}+\frac{t^{4}}{16^{2}}-\frac{t^{6}}{16^{2} \cdot 20}+\frac{11 t^{8}}{16^{4} \cdot 9}-\frac{23 t^{10}}{16^{5} \cdot 150} .
$$

Let

$$
\phi(t)=-\frac{v^{\prime}(t)}{v(t)}-\frac{5}{2 t} .
$$

In particular, from $(3.10),(3.11)$ and the definition of $\phi$ we see that

$$
\phi(2.4) \leq-0.3610558677 \text {. }
$$

It can easily be seen that $\phi$ satisfies the equation

$$
\phi^{\prime}(t)=\phi^{2}(t)+1+v(t)-\frac{15}{4 t^{2}} .
$$

Using the estimate in (3.3) we obtain

$$
1+v(t)-\frac{15}{4 t^{2}} \leq 1+\frac{1}{2} e^{-\frac{t^{2}}{12}}-\frac{15}{4 t^{2}}:=g(t) .
$$

Since $g^{\prime}(t)>0$ for $t \in[0,4.5]$ and $g(4.5) \leq 0.9073055:=a^{2}$, we have

$$
\phi^{\prime}(t) \leq \phi^{2}(t)+a^{2}
$$


for $t \in[0,4.5]$. Thus, integrating (3.18) on $\left[2.4, x_{1}\right]$ we obtain

$$
\frac{1}{a}\left(\frac{\pi}{2}-\arctan \frac{\phi(2.4)}{a}\right) \leq x_{1}-2.4
$$

Hence

$$
x_{1} \geq 4.42940506
$$

where we have also used (3.13) and the definition of $a$.

Now, we estimate $E\left(x_{1}\right)$. From Lemma 2.1 we have

$$
\begin{aligned}
x_{1}{ }^{6} E\left(x_{1}\right) & =\int_{0}^{1.5} r^{5} v^{2}(r) d r+\int_{1.5}^{\sqrt{8}} r^{5} v^{2}(r) d r+\int_{\sqrt{8}}^{x_{1}} r^{5} v^{2}(r) d r \\
& \leq \frac{1.5^{6}}{6 \cdot 2^{2}}+(0.3785)^{2}\left(\frac{8^{3}-1.5^{6}}{6}\right) \\
& +(0.21667)^{2}\left(\frac{x_{1}^{6}-8^{3}}{6}\right),
\end{aligned}
$$

where we have estimated $v(1.5)$ and $v(\sqrt{8})$ by using (3.3). Since $E\left(x_{1}\right)=\left(v^{\prime}\left(x_{1}\right)\right)^{2} / 2$, from (3.19) and (3.20) it follows that

$$
\left|v^{\prime}\left(x_{1}\right)\right| \leq 0.133715,
$$

which together with (3.19) proves the lemma.

Lemma 3.3. Let $\overline{x_{1}}>x_{1}$ be the first zero of $v^{\prime}\left(\cdot, 1, \frac{1}{2}\right)$. Then

$$
5.97 \leq \overline{x_{1}} \leq 6.171
$$

and

$$
\left|v\left(\overline{x_{1}}\right)\right| \leq 0.0712 \text {. }
$$

Proof. Since $\theta^{\prime}(t) \geq 1$, (see $\left.(3.6)\right)$ on $\left[x_{1}, \overline{x_{1}}\right]$ we see that $\overline{x_{1}}-x_{1} \leq \frac{\pi}{2}$. Hence

$$
\overline{x_{1}} \leq x_{1}+\frac{\pi}{2} \leq 6.1707963 .
$$

Furthermore, from (3.21) and (3.22) we infer

$$
1+|v(t)|-\frac{15}{4 t^{2}} \leq 1+\left|v^{\prime}\left(x_{1}\right)\right|-\frac{15}{{\overline{x_{1}}}^{2}} \leq 1.0352348
$$

Thus, arguing as in (3.16) we obtain

$$
\overline{x_{1}} \geq x_{1}+\frac{\pi}{2 \sqrt{1.0352348}} \geq 5.973284 .
$$

In order to estimate $v\left(x_{1}\right)$ we use Lemma 2.1. Since $v^{\prime}\left(\overline{x_{1}}\right)=0$, we have

$$
{\overline{x_{1}}}^{6}\left(\frac{v^{2}\left(\overline{x_{1}}\right)}{2}+\frac{v^{3}\left(\overline{x_{1}}\right)}{3}\right) \leq \frac{\left(v^{\prime}\left(x_{1}\right)\right)^{2}}{2} x_{1}{ }^{6}+\int_{x_{1}}^{\overline{x_{1}}} r^{5} v^{2}(r) d r .
$$

Hence

$$
v^{2}\left(\overline{x_{1}}\right) \leq \frac{3\left(v^{\prime}\left(x_{1}\right)\right)^{2}}{2\left(\frac{\overline{x_{1}}}{x_{1}}\right)^{6}+1} \leq(0.0711735)^{2},
$$

where we have used (3.19) and (3.22). This together with (3.22) and (3.24) proves the lemma. 
Lemma 3.4. If $x_{2}$ is the second zero of $v\left(\cdot, 1, \frac{1}{2}\right)$, then

$$
7.5 \leq x_{2} \leq 8.1
$$

and

$$
v^{\prime}\left(x_{2}\right) \leq 0.0612 \text {. }
$$

Proof. Let $w(t):=t^{\frac{5}{2}} v(t)$. It can easily be shown that $w$ satisfies

$$
w^{\prime \prime}(t)+\left(1-\frac{15}{4 t^{2}}+|v(t)|\right) w(t)=0 .
$$

Since

$$
1-\frac{15}{4 t^{2}}+|v(t)| \geq 1-\frac{15}{4 x_{1}^{2}} \geq .8088
$$

(see (3.19)), from the Sturm comparison theorem and Lemma 3.1 we infer

$$
x_{2} \leq x_{1}+\frac{\pi}{\sqrt{.8088}} \leq 8.0931 .
$$

On the other hand, for $t \in\left[x_{1}, x_{2}\right]$ using (3.25) and (3.27) we have

$$
1-\frac{15}{4 t^{2}}+|v(t)| \leq 1-\frac{15}{4 x_{2}^{2}}+\left|v\left(\overline{x_{1}}\right)\right| \leq 1.0139201 .
$$

Hence,

$$
x_{2} \geq x_{1}+\frac{\pi}{\sqrt{1.0139201}} \geq 7.5494033,
$$

where we have also used (3.19).

Next, from (3.28), Lemma 3.1 and Lemma 3.2 we see that

$$
\left(v^{\prime}\left(x_{2}\right)\right)^{2} \leq \frac{\left(v^{\prime}\left(x_{1}\right)\right)^{2}}{6}\left\{5\left(\frac{4.6}{7.549}\right)^{6}+1\right\} \leq(.061175)^{2},
$$

which together with (3.27) and (3.28) concludes the proof of the lemma.

Lemma 3.5. Let $x_{3}$ denote the third zero of $v\left(\cdot, 1, \frac{1}{2}\right)$. Then

$$
10.66 \leq x_{3} \leq 11.35,
$$

and

$$
\left|v^{\prime}\left(x_{3}\right)\right| \leq 0.035
$$

Proof. Since on $\left[x_{2}, x_{3}\right]$ we have

$$
1-\frac{15}{4 t^{2}}+|v(t)| \geq 1-\frac{15}{4 x_{2}^{2}} \geq .9325697,
$$

by the Sturm comparison theorem and (3.28) we see that

$$
x_{3} \leq x_{2}+\frac{\pi}{\sqrt{.9325697}} \leq 11.346371 .
$$

On the other hand, imitating the arguments of Lemma 3.3 it can easily be shown that if $\overline{x_{2}}>x_{2}$ is such that $v^{\prime}\left(\overline{x_{2}}\right)=0$, then $v\left(\overline{x_{2}}\right) \leq 0.0476045$. Thus

$$
1-\frac{15}{4 t^{2}}+|v(t)| \leq 1.04761-\frac{15}{4 x_{3}^{2}} \leq 1.0184761 .
$$


Hence,

$$
x_{3} \geq x_{2}+\frac{\pi}{\sqrt{1.0184761}} \geq 10.66237,
$$

where we have also used (3.28). Furthermore,

$$
\left(v^{\prime}\left(x_{3}\right)\right)^{2} \leq \frac{\left(v^{\prime}\left(x_{2}\right)\right)^{2}}{6}\left\{5\left(\frac{8.0932}{10.66}\right)^{6}+1\right\} \leq(.035)^{2} .
$$

In particular, from (3.30) and (3.31) we see that

$$
\left|v^{\prime}\left(x_{3}\right)\right| \leq \frac{4.5}{x_{3}^{2}} \text {. }
$$

Thus, the lemma is proven.

\section{The First Four Zeroes of $v_{d}\left(\cdot, 1, \frac{1}{2}\right)$}

In this section we estimate the first four zeros of $v_{d}$, where $v_{d}$ denotes the partial derivative of $v$ with respect to $d$.

Lemma 4.1. Let $\tau_{1}$ denote the first zero of $v_{d}\left(\cdot, 1, \frac{1}{2}\right)$. Then $\tau_{1} \geq 4.04$.

Proof. Since $v_{d}(0)=1$ and $v(0)=\frac{1}{2}$ we have

$$
-v_{d}^{\prime}(t)=t^{-5} \int_{0}^{t} r^{5}(1+2|v(r)|) v_{d}(r) d r \leq \frac{t}{3}
$$

for $t \in\left[0, \tau_{1}\right]$. Thus

$$
v_{d}^{\prime}(t) \geq 1-\frac{t^{2}}{6}
$$

On the other hand, using (3.9) and (4.2) in (4.1) we infer

$$
-v_{d}^{\prime}(t) \geq t^{-5} \int_{0}^{t} r^{5}\left(1+1-\frac{r^{2}}{8}\right)\left(1-\frac{r^{2}}{6}\right) d r=\frac{t}{3}\left(1-\frac{11 t^{2}}{64}+\frac{t^{4}}{160}\right) .
$$

Integrating (4.3) we obtain

$$
v_{d}(t) \leq 1-\frac{t^{2}}{2}+\frac{11 t^{4}}{768}-\frac{t^{6}}{2880},
$$

for $t \in[0, \sqrt{6}]$. Reiterating the argument and using (3.11) and (4.4) in (4.1) we see that

$$
\begin{aligned}
-v_{d}^{\prime}(t) & \leq t^{-5} \int_{0}^{t} r^{5}\left(2-\frac{r^{2}}{8}+\frac{r^{4}}{128}\right)\left(1-\frac{r^{2}}{6}+\frac{11 r^{4}}{768}\right) d r \\
& \leq \frac{t}{3}\left(1-\frac{11 t^{2}}{64}+\frac{11 t^{4}}{640}-\frac{19 t^{6}}{16^{2} \cdot 6}+\frac{11 t^{8}}{16^{2} \cdot 112}\right),
\end{aligned}
$$

and

$$
v_{d}(t) \geq 1-\frac{t^{2}}{2}+\frac{11 t^{4}}{12 \cdot 64}-\frac{11 t^{6}}{18 \cdot 640}+\frac{19 t^{8}}{16^{2} \cdot 144}-\frac{11 t^{10}}{64^{3} \cdot 560}
$$

Let

$$
\psi(t)=-\frac{v_{d}^{\prime}(t)}{v_{d}(t)}-\frac{5}{2 t}
$$


In particular, from (4.5), (4.6) and the definition of $\psi$ we see that

$$
\psi(1.8) \leq-0.2597377 .
$$

It can easily be seen that $\psi$ satisfies the equation

$$
\psi^{\prime}(t)=\psi^{2}(t)+1+2 v(t)-\frac{15}{4 t^{2}} .
$$

From the estimate for $v$ on $[0, \sqrt{8}]$ (see $(3.11)$ ) we obtain

$$
1+2 v(t)-\frac{15}{4 t^{2}} \leq 1-\frac{t^{2}}{8}+\frac{t^{4}}{128}-\frac{t^{6}}{7680}-\frac{15}{4 t^{2}}:=G(t) .
$$

Now, we calculate the maximum of $G(t)$ on $[0, \sqrt{8}]$. Since

$$
G^{\prime}(t)=-\frac{1}{2 t^{3}}\left(\frac{t^{4}}{2}\left(1-\frac{t^{2}}{8}+\frac{t^{4}}{320}\right)-15\right) .
$$

we see that $1-\frac{t^{2}}{8}+\frac{t^{4}}{320}$ has its minimum at $t=\sqrt{20}$. For $t \in[2,2.4]$ we have that $\frac{t^{4}}{2}-\frac{t^{6}}{16}+\frac{t^{8}}{640}-15 \leq 0$, hence $G^{\prime}(t) \geq 0$. Also, for $t \in[0,2]$ we see that $\frac{t^{4}}{2}+\frac{t^{8}}{640}-15 \leq 0$. Therefore $G^{\prime}(t) \geq 0$ for $t \in[0,2.4]$. Thus, $G(t) \leq G(2.4) \leq 0.8632751:=b^{2}$. Hence, for $t \in[1.8,2.4]$ we have

$$
\psi^{\prime}(t) \leq \psi^{2}(t)+b^{2} .
$$

Furthermore, by integrating (4.11) on $[1.8,2.4]$ and using $(4.9)$ we obtain

$$
\frac{1}{b}\left(\arctan \frac{\psi(2.4)}{b}-\arctan \frac{\psi(1.8)}{b}\right) \leq 0.6 \text {. }
$$

Thus,

$$
\psi(2.4) \leq-0.1254803 .
$$

For $t>2.4$ with $v(t)>0$, using (3.3) we infer

$$
1+2 v(t)-\frac{15}{4 t^{2}} \leq 1+e^{-\frac{t^{2}}{12}}-\frac{15}{t^{2}}:=G_{1}(t) .
$$

Since

$$
G_{1}^{\prime}(t)=-\frac{t}{6} e^{-\frac{t^{2}}{12}}-\frac{15}{2 t^{3}}
$$

we see that $\max G_{1}(t)=1.0597904=G_{1}(3.2)$, hence by integrating

$$
\psi^{\prime}(t) \leq \psi^{2}(t)+G_{1}(3.2)
$$

on $[2.4, t]$ we see that if $v_{d}\left(\tau_{1}\right)=0$ then

$$
\frac{1}{\sqrt{G_{1}(3.2)}}\left(\frac{\pi}{2}-\arctan \frac{\psi(2.4)}{\sqrt{G_{1}(3.2)}}\right) \leq \tau_{1}-2.4 .
$$

Thus

$$
\tau_{1} \geq 4.043663
$$

where we have also used (4.13). Hence, the lemma is proven.

Lemma 4.2. If $\tau_{2}$ is the second zero of $v_{d}\left(\cdot, 1, \frac{1}{2}\right)$, then

$$
\tau_{2} \geq 7.059 \text {. }
$$


Proof. In order to estimate $\tau_{2}$ we note that $z(t):=t^{\frac{5}{2}} v_{d}(t)$ satisfies

$$
z^{\prime \prime}(t)+\left(1-\frac{15}{4 t^{2}}+2|v(t)|\right) z(t)=0 .
$$

First we estimate $v$ on $\left[\tau_{1}, x_{1}\right]$. Since

$$
v^{\prime \prime}(t)=-\frac{5}{t} v^{\prime}(t)-|v(t)| v(t) \leq-\frac{5}{t} v^{\prime}(t),
$$

after integration on $\left[\tau_{1}, x_{1}\right]$ we infer

$$
v^{\prime}(t) \geq v^{\prime}\left(x_{1}\right) \frac{x_{1}^{5}}{t^{5}} .
$$

Hence

$$
v\left(x_{1}\right)-v\left(\tau_{1}\right) \geq \frac{v^{\prime}\left(x_{1}\right) x_{1}^{5}}{4}\left(\tau_{1}^{-4}-x_{1}^{-4}\right) .
$$

Therefore,

$$
v\left(\tau_{1}\right) \leq 0.1046821
$$

Hence on $\left[\tau_{1}, x_{1}\right]$ we have

$$
1-\frac{15}{4 t^{2}}+2|v(t)| \leq 1-\frac{15}{4 x_{1}^{2}}+2 v\left(\tau_{1}\right) \leq 1.032143 .
$$

For $t>x_{1}$ by using (3.19) we obtain

$$
1-\frac{15}{4 t^{2}}+2|v(t)| \leq 1-\frac{15}{4 x_{1}^{2}}+2 v\left(\overline{x_{1}}\right) \leq 1.0850936 .
$$

Thus by the Sturm comparison theorem from (4.20)-(4.21) using also (4.15) we see that

$$
\tau_{2} \geq \tau_{1}+\frac{\pi}{\sqrt{1.0850936}} \geq 7.0595584
$$

which concludes the proof of the lemma.

Lemma 4.3. Let $\tau_{3}<\tau_{4}$ denote the third zero and the fourth zero of $v_{d}\left(\cdot, 1, \frac{1}{2}\right)$. Then

$$
\tau_{3} \geq 10.07
$$

and

$$
\tau_{4} \geq 13.09 \text {. }
$$

Proof. Since on $\left[\tau_{2}, x_{2}\right]$ we know that $\left|v\left(\tau_{2}\right)\right| \leq\left|v\left(\overline{x_{1}}\right)\right|$ it follows that

$$
1-\frac{15}{4 t^{2}}+2|v(t)| \leq 1-\frac{15}{4 x_{2}^{2}}+2\left|v\left(\overline{x_{1}}\right)\right| \leq 1.0850936 .
$$

On the other hand, for $t>x_{2}$ we have

$$
1-\frac{15}{4 t^{2}}+2|v(t)| \leq 1-\frac{15}{4 x_{3}^{2}}+2\left|v\left(\overline{x_{1}}\right)\right| \leq 1.0660916 .
$$

Hence,

$$
\tau_{3} \geq \tau_{2}+\frac{\pi}{\sqrt{1.0850936}} \geq 10.075454
$$


Similarly

$$
\tau_{4} \geq \tau_{3}+\frac{\pi}{\sqrt{1.0850936}} \geq 13.091349
$$

Thus, the lemma is proven.

\section{Proof of Theorem 1.2}

In order to prove the separation of zeroes for $v$ and $v_{d}$ we first show

Lemma 5.1. If $x_{i}$ and $x_{i+1}$ are two consecutive zeroes of $v\left(\cdot, 1, \frac{1}{2}\right)$ on $\left[x_{3}, \infty\right)$, then $x_{i+1}-x_{i} \leq 3.46$. Moreover,

$$
\frac{x_{i}}{x_{i+1}} \geq .75 .
$$

Proof. Without loss of generality we can assume that $v^{\prime}\left(x_{i}\right)>0$. Let $\bar{x}_{i} \in\left(x_{i}, x_{i+1}\right)$, $i \geq 3$, be such that $v^{\prime}\left(\bar{x}_{i}\right)=0$. Then, from (3.6) we see that $\theta^{\prime} \geq 1$ on $\left[x_{i}, \bar{x}_{i}\right]$. Thus

$$
\bar{x}_{i}-x_{i} \leq \frac{\pi}{2} \text {. }
$$

On the other hand on $\left[\bar{x}_{i}, x_{i+1}\right]$, using the fact that $x_{i} \geq 10$ we infer

$$
\theta^{\prime}(t) \geq 1-\frac{5}{2 t} \sin 2 \theta \geq 1-.25 \sin 2 \theta \text {. }
$$

Hence, by integrating (5.2) we obtain

$$
\begin{aligned}
x_{i+1}-\bar{x}_{i} & \leq \int_{\frac{\pi}{2}}^{\pi} \frac{d \theta}{1-.25 \sin 2 \theta}=\int_{0}^{\frac{\pi}{2}} \frac{d y}{1-.25 \sin y}=\frac{2}{\sqrt{1-\frac{1}{16}}} \arctan \frac{1.25}{\sqrt{1-\frac{1}{16}}} \\
& \leq 1.8833 .
\end{aligned}
$$

Therefore, combining (5.1) with (5.3) we have

$$
x_{i+1}-x_{i} \leq 3.455 .
$$

Furthermore,

$$
\frac{x_{i}}{x_{i+1}} \geq \frac{x_{i}}{x_{i}+3.455} \geq .7524
$$

which proves the lemma.

Lemma 5.2. Let $x_{3}<x_{4}<\ldots$ denote the zeroes of $v\left(\cdot, 1, \frac{1}{2}\right)$ on $[10, \infty)$. If $\left|v^{\prime}\left(x_{3}\right)\right| \leq \frac{M}{x_{3}^{2}}$ then

$$
\left|v^{\prime}\left(x_{i}\right)\right| \leq \frac{M}{x_{i}^{2}} .
$$

Proof. We prove the latter inequality by induction. As shown in (3.32) it holds for $i=3$. Suppose $\left|v^{\prime}\left(x_{i}\right)\right| \leq \frac{M}{x_{i}^{2}}$. By Lemma 5.1 we have

$$
\frac{5}{6}\left(\frac{x_{i}}{x_{i+1}}\right)^{6}+\frac{1}{6} \leq \frac{x_{i}^{4}}{\left(x_{i+1}\right)^{4}}
$$

because $\left(\frac{x_{i}}{x_{i+1}}\right)^{2} \geq .558$. Hence, by $(2.1)$ we see that $\left|v^{\prime}\left(x_{i}\right)\right| \leq \frac{M}{x_{i+1}^{2}}$, which concludes the proof of the lemma. 
Lemma 5.3. For any $j \geq 3$ the following holds

$$
x_{j} \leq x_{3}+(j-3) \pi+.68
$$

and

$$
\tau_{j+1} \geq \tau_{4}+(j-3) \pi-.23 .
$$

Proof. Since for $t \in\left[x_{i}, x_{i+1}\right]$ we have

$$
1-\frac{15}{4 t^{2}}+|v(t)| \geq 1-\frac{15}{4 x_{i}^{2}}
$$

by the Sturm comparison theorem it follows that

$$
\begin{aligned}
x_{i+1} & \leq x_{i}+\frac{\pi}{\sqrt{1-\frac{15}{4 x_{i}^{2}}}} \leq x_{i}+\frac{\pi}{1-\frac{15}{4 x_{i}^{2}}} \\
& =x_{i}+\pi\left(1+\frac{15}{4 x_{i}^{2}}+\left(\frac{15}{4 x_{i}^{2}}\right)^{2}+\cdots\right) \\
& =x_{i}+\pi+\frac{15 \pi}{4 x_{i}^{2}} \cdot \frac{1}{1-\frac{15}{4 x_{i}^{2}}} .
\end{aligned}
$$

Since $x_{i} \geq 10.66$ we obtain

$$
x_{i+1} \leq x_{i}+\pi+\frac{15}{4 x_{i}^{2}} \cdot \frac{400}{385} \leq x_{i}+3.25 .
$$

On the other hand using Lemma 4.2 we see that

$$
1-\frac{15}{4 t^{2}}+|v(t)| \leq 1-\frac{15}{4 x_{i+1}^{2}}+\frac{M}{x_{i}^{2}} \leq 1+\frac{M}{x_{i}^{2}} .
$$

Hence, from the Sturm comparison theorem it follows that

$$
\begin{aligned}
x_{i+1} & \geq x_{i}+\frac{\pi}{\sqrt{1+\frac{M}{x_{i}^{2}}}} \geq x_{i}+\frac{\pi}{1+\frac{M}{2 x_{i}^{2}}} \\
& =x_{i}+\pi\left(1-\frac{M}{2 x_{i}^{2}}+\left(\frac{M}{2 x_{i}^{2}}\right)^{2}-\cdots\right) \\
& =x_{i}+\pi-\frac{\pi M}{2 x_{i}^{2}} \cdot \frac{1}{1+\frac{M}{2 x_{i}^{2}}} \\
& \geq x_{i}+\pi-.12 \geq x_{i}+3,
\end{aligned}
$$

where we have used the fact that $x_{i} \geq 10$ and $M=4.5$ (see (3.32)). Thus,

$$
x_{i+1} \geq x_{3}+3(i-3) \text {. }
$$

Hence

$$
\begin{aligned}
\frac{400}{385} \sum_{j=3}^{n} \frac{15 \pi}{4\left(x_{3}+3(j-3)\right)^{2}} & \leq \frac{400}{385} \cdot \frac{15 \pi}{4} \cdot \frac{1}{3} \int_{-3}^{\infty} \frac{d s}{\left(x_{3}+3 s\right)^{2}} \\
& \leq \frac{400}{385} \cdot \frac{15 \pi}{4 \cdot 18} \leq .68 .
\end{aligned}
$$

Similarly

$$
\tau_{i+1} \leq \tau_{i}+3.2
$$


and

$$
\tau_{i+1} \geq \tau_{i}+\pi-\frac{M \pi}{\tau_{i}^{2}}(.96) \geq x_{i}+2.9
$$

Hence

$$
\begin{aligned}
\sum_{j=4}^{n} \frac{M \pi(.96)}{\left(\tau_{4}+(j-4) 2.9\right)^{2}} & \leq \frac{M \pi(.96)}{2.9} \int_{-4}^{\infty} \frac{d s}{\left(\tau_{4}+2.9 s\right)^{2}} \\
& \leq \frac{M \pi(.96)}{2.9^{2} \tau_{4}} \leq \frac{M \pi(.96)}{2.9^{2}} \cdot \frac{1}{13} \leq .23 .
\end{aligned}
$$

Thus the lemma is proven.

By the Sturm comparison theorem we see that $\tau_{j}<x_{j}$ for $j=1,2, \ldots$ On the other hand, by Lemmas 3.5, 4.3 and 5.3 we have

$$
\begin{aligned}
x_{j} & \leq x_{3}+(j-3) \pi+.68 \\
& \leq \tau_{j+1}-\tau_{4}+x_{3}+.68+.23 \\
& \leq \tau_{j+1}-13.09+11.35+.68+.23 \\
& <\tau_{j+1} .
\end{aligned}
$$

Hence, from (5.15) and the results of sections 3 and 4 on the first three zeroes of $v$ and the first four zeroes of $v_{d}$ we see that for $j=1,2, \ldots$ we have

$$
\tau_{j}<x_{j}<\tau_{j+1}<x_{j+1} .
$$

Thus, the zeroes of $v\left(\cdot, 1, \frac{1}{2}\right)$ separate the zeroes of $v_{d}\left(\cdot, 1, \frac{1}{2}\right)$ and conversely, which concludes the proof of Theorem 1.2.

\section{Proof of Theorem 1.1}

The crucial ingredient in the proof of Theorem 1.1 is Lemma 6.3 below showing that the zeroes of $v(t, \lambda, d)$ separate the zeros of $v_{d}(t, \lambda, d)$.

It can easily be shown that the following homogeneity relation holds

$$
v\left(t, 1, \frac{d}{\lambda}\right)=\frac{1}{\lambda} v\left(\frac{t}{\sqrt{\lambda}}, \lambda, d\right) .
$$

Let $t_{j}(\lambda, d)$ denote the $j$-th zero of $v(\cdot, \lambda, d)$ and $\tau_{j}(\lambda, d)$ be the $j$-th zero of $v_{d}(\cdot, \lambda, d)$. From (6.1) we see that

$$
t_{j}(\lambda, d)=\sqrt{\lambda} t_{j}\left(1, \frac{d}{\lambda}\right)
$$

and

$$
\tau_{j}(\lambda, d)=\sqrt{\lambda} \tau_{j}\left(1, \frac{d}{\lambda}\right)
$$

Using (6.1) and the results of [1] we have

$$
\lim _{d \rightarrow \infty} t_{j}(\lambda, d)=\sqrt{\lambda} t_{j}\left(1, \frac{d}{\lambda}\right)
$$

and

$$
\lim _{d \rightarrow \infty} t_{1}(\lambda, d)=0,
$$

for $\lambda$ in compact subsets of $(0, \infty)$. 
Lemma 6.1. Let $s_{1}:=s_{1}(d)$ denote the first zero of $v^{\prime}(\cdot, 1, d)$. Then $s_{1} \rightarrow 0$, as $d \rightarrow \infty$.

Proof. For $t \leq s_{1}$ we have

$$
\begin{aligned}
-t^{5} v^{\prime}(t) & =\int_{0}^{t} r^{5}(1+|v(r)|) v(r) d r \\
& =\int_{0}^{t_{1}} r^{5}(1+|v(r)|) v(r) d r+\int_{t_{1}}^{t} r^{5}(1+|v(r)|)(-v(r)) d r .
\end{aligned}
$$

Also, from Lemma 2.1 we have

$$
t^{6} \frac{\left(v^{\prime}(t)\right)^{2}}{2}+2 t^{5} v(t) v^{\prime}(t)+t^{6}\left(\frac{|v(t)|^{3}}{3}+\frac{\lambda v^{2}(t)}{2}\right) \geq 0 .
$$

Hence, using the quadratic formula we see that

$$
2 t^{10} v^{2}(t) \geq t^{12}\left(\frac{|v(t)|^{3}}{3}+\frac{\lambda v^{2}(t)}{2}\right) \geq t^{12} \frac{|v(t)|^{3}}{3} .
$$

Thus,

$$
|v(t)| \leq 6 t^{-2}
$$

for $t \in\left[0, t_{1}\right]$.

Since, by the results from [1] we know that $t_{1}(1, d) \rightarrow 0$ as $d \rightarrow \infty$ and since (6.7) holds, we have

$$
\int_{0}^{t} r^{5}(1+|v(r)|) v(r) d r \leq 6\left(\frac{t_{1}^{4}}{4}+6 \frac{t_{1}^{2}}{2}\right) \rightarrow 0,
$$

as $d \rightarrow \infty$.

Suppose $s_{1}$ is bounded away from zero, hence there exists $m>0$ such that

$$
s_{1}>m \text {. }
$$

Let $0<y<\min \left\{t_{1}\left(1,-\frac{1}{2}\right), m\right\}$. On $\left[t_{1}, s\right]$ we see that $v^{\prime \prime}=-\frac{5}{r} v^{\prime}-v-|v| v \geq 0$. Hence, since $v$ is convex on $\left[t_{1}, s\right]$ and since for $d$ large $v(\cdot, 1, d) \rightarrow v\left(\cdot, 1,-\frac{1}{2}\right)$ we have

$$
\begin{aligned}
\int_{t_{1}}^{t} r^{5}(1+|v(r)|) v(r) d r & \geq \int_{t_{1}}^{t} r^{5} \frac{v(y)}{y-t_{1}}\left(r-t_{1}\right) d r \\
& \geq K|v(y)|\left(\frac{t^{7}}{7}+\frac{t_{1}^{7}}{6}\right),
\end{aligned}
$$

where $K$ is a constant. Since $t_{1} \rightarrow 0$, as $d \rightarrow \infty$, from (6.6), (6.8) and (6.10) with $t=s_{1}$ we get a contradiction, which proves the lemma.

Lemma 6.2. If $\tau_{j}(\lambda, d)$ denotes the $j$-th zero of $v_{d}(\cdot, \lambda, d)$, then

$$
\lim _{d \rightarrow \infty} \tau_{j}(\lambda, d)=\sqrt{\lambda} \tau_{j-1}\left(1,-\frac{1}{2}\right),
$$

and 


$$
\lim _{d \rightarrow \infty} \tau_{1}(\lambda, d)=0
$$

for $\lambda$ in compact subsets of $(0, \infty)$.

Proof. Let $\sigma_{1}:=\sigma_{1}(d)$ denote the first zero of $v_{d}^{\prime}(\cdot, 1, d)$. By the Sturm comparison theorem and Lemma 6.1 we know that $\sigma_{1}<s_{1}$. Let $w$ be the multiple of $v_{d}(\cdot, 1, d)$ that satisfies

$$
\begin{aligned}
w^{\prime \prime}(t)+\frac{5}{t} w^{\prime}(t)+w(t)+2 w(t)|v(t, 1, d)| & =0, \quad t \in\left[\sigma_{1}, \infty\right), \\
w\left(\sigma_{1}\right) & =0, \\
w^{\prime}\left(\sigma_{1}\right) & =-1 .
\end{aligned}
$$

Since $v_{d}\left(\cdot, 1,-\frac{1}{2}\right)=-z$ satisfies

$$
z^{\prime \prime}(t)+\frac{5}{t} z^{\prime}(t)+z(t)+2 z(t)\left|z\left(t, 1,-\frac{1}{2}\right)\right|=0,
$$

and

$$
\left|z^{\prime}(t)\right| \leq \frac{t}{3}
$$

as $d \rightarrow \infty$, we have

$$
\left|z\left(\sigma_{1}\right)+1\right| \leq \frac{\sigma_{1}^{2}}{6} \rightarrow 0
$$

Thus by the continuous dependence on initial conditions and coefficients we see that $w(\cdot, 1, d)$ converges to $z\left(\cdot, 1,-\frac{1}{2}\right)$ uniformly on compact subsets of $[0, \infty)$ as $d \rightarrow \infty$. Since the zeros of $w(\cdot, 1, d)$ are the zeros of $v_{d}(\cdot, 1, d)$ we see that $\tau_{j}(1, d) \rightarrow \tau_{j-1}\left(1,-\frac{1}{2}\right)$ as $d \rightarrow \infty$. Hence the lemma follows from (6.3) and the Sturm comparison theorem.

Lemma 6.3. For $d$ sufficiently large and for $\lambda$ in compact subsets of $(0, \infty)$, the zeroes of $v(\cdot, \lambda, d)$ separate the zeroes of $v_{d}(\cdot, \lambda, d)$. Moreover,

$$
v^{\prime}(1, \lambda, d) \cdot v_{d}(1, \lambda, d)>0 .
$$

Proof. The separation of zeroes follows from the Sturm comparison theorem, Lemma 6.2 and Theorem 1.2. Hence

$$
\tau_{1}(\lambda, d)<t_{1}(\lambda, d)<\ldots<\tau_{k}<t_{k}=1<\tau_{k+1} .
$$

Also, because $v_{d}^{\prime}\left(\tau_{1}\right)<0$ we have $\operatorname{sgn} v_{d}^{\prime}\left(\tau_{i}\right)=(-1)^{i+1}$. Hence $v\left(\tau_{i}\right) v_{d}^{\prime}\left(\tau_{i}\right)<0$. Finally, if $v^{\prime}(1)>0$, then $v<0$ on $\left(t_{k-1}, t_{k}=1\right)$. Thus, $v_{d}^{\prime}\left(\tau_{j-1}\right)>0$. Hence $v_{d}(1)>0$. Similarly, if $v^{\prime}(1)<0$, then $v_{d}(1)<0$, which concludes the proof of the lemma.

Next, we recall various properties of the bifurcation diagram of the set of radial solutions to (1.1). We summarize these properties in the following lemma.

Lemma 6.4. a) $\Gamma$ is a connected component of $\{(\lambda, d) ; v(1, \lambda, d)=0\}$ if and only if there exists a positive integer $k$ and a continuous function $\beta_{k}:(0, \infty) \rightarrow(0, \infty)$ such that $\Gamma=\left\{\left(\beta_{k}(d), \beta_{k}(d), d\right) ; d \in(0, \infty)\right\}:=\Gamma_{k}$. 
b) If $\left\{\left(\lambda_{n}, d_{n}\right)\right\}$ is a sequence in $\Gamma_{k}$ with $d_{n} \rightarrow \infty$ then $\left\{\lambda_{n}\right\}$ converges to $t_{k-1}\left(1,-\frac{1}{2}\right)$.

c) If $(\lambda, d) \in \Gamma_{k}$ then $\lambda<\mu_{k}$, where $\mu_{k}$ is a radial eigenvalue of $-\Delta$ subject to a zero Dirichlet boundary condition.

d) For each $k \geq 1$ there exists $q(k)>0$ such that if $(\lambda, d) \in \Gamma_{k}$ then $\lambda \geq q(k)$ and $q(k) \rightarrow \infty$ as $k \rightarrow \infty$.

e) $\{(\lambda, d) ; v(1, \lambda, d)=0, v(\cdot, \lambda, d)$ has exactly $k$ zeroes in $(0,1]\}$ is connected.

f) For each positive integer $k$ there exist a positive number $r$ and a differentiable decreasing function $s:(r, \infty) \rightarrow(0, \infty)$ such that $(\lambda, d) \in \Gamma_{k}$ if and only if $\lambda=s(d)$.

Proof. The proof of parts a)-e) is identical to those in [5, Lemma 5.2]. Part f) follows from the implicit function theorem, Lemma 6.3 and part b).

Now, we conclude the proof of Theorem 1.1 .

From part d) of Lemma 6.4 we see that for fixed $\lambda>0$ there can not exist radial solutions with arbitrarily large number of zeros. This proves the existence of $j(\lambda)$. Also if $\lambda \neq t_{i}\left(1,-\frac{1}{2}\right), i=1,2, \ldots$, then the existence of $D(\lambda)$ follows from parts d) and b) of Lemma 6.4.

Suppose, now, that $\lambda=t_{i}$ for positive integer $i$ and that $v\left(1, \lambda, d_{n}\right)=0$ for some sequence $\left\{d_{n}\right\} \rightarrow \infty$. By part $\mathrm{d}$ ) of Lemma 6.4 , without loss of generality we can assume that $v\left(\cdot, \lambda, d_{n}\right)$ has $j$ zeroes in $(0,1]$. Thus, by parts a) and e) of Lemma 6.4 we see that $\left\{\left(\lambda, d_{n}\right)\right\} \subset \Gamma_{j}$. Since, by Lemma 6.4 part f), $\lambda=s\left(d_{n}\right)$ for $n$ large we have a contradiction to the fact that $s$ is decreasing. Thus, the existence of $D(\lambda)$ for all $\lambda>0$ is established.

Part b) of Theorem 1.1 follows from part f) of Lemma 6.4. Hence, Theorem 1.1 is proven.

\section{REFERENCES}

1. F. Atkinson, H. Brezis, L. Peletier, Solutions d'equations elliptiques avec exposant de Sobolev critique qui changent de signe, C. R. Acad. Sci. Paris, t. 306, Serie I (1988), 711-714. MR 89k:35088

2. H. Brezis, L. Nirenberg, Positive solutions of nonlinear elliptic equations involving critical Sobolev exponents, Comm. Pure Appl. Math. 36(1983), 437-477. MR 84h:35059

3. A. Castro, A. Kurepa, Infinitely many radially symmetric solutions to a superlinear Dirichlet problem in a ball, Proc. Amer. Math. Soc. 101(1987), 56-64. MR 88j:35058

4. __ Radially symmetric solutions to a superlinear Dirichlet problem in a ball with jumping nonlinearities, Trans. Amer. Math. Soc. 315(1989), 353-372. MR 90g:35053

5. __ Radially symmetric solutions to a Dirichlet problem involving critical exponents, Trans. Amer. Math. Soc. 343(1994), 907-926. MR 94h:35012

6. G. Cerami, Elliptic equations with critical growth, College on Variational Problems in Analysis, Lecture Notes SMR 281/24, International Centre for Theoretical Physics, Trieste, Italy, (1988).

7. G. Cerami, S. Solimini, M. Struwe, Some existence results for superlinear elliptic boundary value problems involving critical exponents, J. Functional Anal. 69(1986), 289-306. MR 88b:35074

8. M. G. Crandall, P. M. Rabinowitz, Bifurcation from simple eigenvalues, J. Functional Analysis 8(1971), 321-340. MR 44:5836

9. M. K. Kwong, Uniqueness of positive solutions for $\Delta u-u+u^{p}=0$ in $R^{n}$, Arch. of Rational Mech. 105 (1989), 243-266. MR 90d:35015

10. S. I. Pohozaev, Eigenfunctions of the equation $\Delta u+\lambda f(u)=0$, Soviet. Math. Dokl. 6(1965), 1408-1411. MR 33:411 
11. P. Pucci, J. Serrin, A general variational identity, Indiana Univ. Math. J. 35(1986), 681-703. MR 88b:35072

12. S. Solimini, On the existence of infinitely many radial solutions for some elliptic problems, Revista Mat. Aplicadas 9 (1987), 75-86. MR 89f:35086

13. N. Trudinger, Remarks concerning the conformal deformation of Riemannian structures on compact manifolds, Ann. Sc. Norm. Sup. Pisa 22(1968), 265-274. MR 39:2093

Department of Mathematics, University of North Texas, Denton, Texas 76203-5116

E-mail address: acastro@unt.edu

Department of Mathematics, North Carolina A\&t State University, Greensboro, North Carolina 27411

E-mail address: kurepaa@athena.ncat.edu 